\title{
Methods of Determining the Beat Length of Planar Waveguides
}

\begin{abstract}
K. GuT*
Department of Optoelectronics, Silesian University of Technology, Akademicka 2A, 44-100 Gliwice, Poland

The paper presents two methods of determining the planar waveguide birefringence and the measuring stands, which are used to determine the beat length of planar waveguide structures. The light is introduced into the waveguide through a prism coupler. The first method applies measurements of scattered light. The second method uses an immersion coupler. The most fundamental property of an immersion coupler is the possibility to change fluently the propagation length while immersing the waveguide.
\end{abstract}

DOI: 10.12693 /APhysPolA.124.425

PACS: 42.25.Ja, 42.25.Lc, 42.25.Fx

\section{Introduction}

In recent years sensors have been developed which do apply various optical phenomena for the purpose of monitoring physical, chemical and biological values [1-6]. Basing on interferential phenomena, extremely sensitive sensors could be constructed [7]. The waveguide technique permits miniature many sensor structure. Due to their simple structure a differential interferometer is being developed [8], whose principle of operation can be seen in Fig. 1.

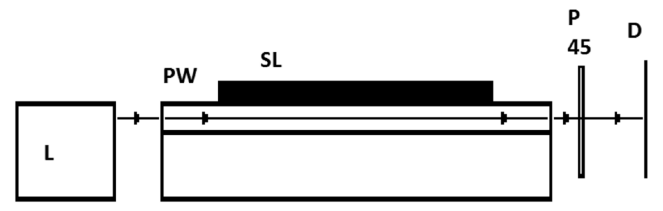

Fig. 1. Diagram of the differential interferometer.

The light from the laser $\mathrm{L}$ is introduced into the planar waveguides $\mathrm{PW}$ in order to excite orthogonal pair of the mode TE and TM. Upon the surface of the waveguide a sensor layer SL can be deposited, which affected by external agents changes its optical or geometrical parameters, resulting in change of a phase velocity of the modes. At the output of the structure (after the bringing of light from the mode TE and TM into one polarization plane through the polarizer $\mathrm{P}$ ) the detector $\mathrm{D}$ records the interferential signal depending on the phase difference between the modes. The most important parameter in the differential interferometer, describing the waveguide is the beat length [9]. Orthogonal modes propagating in a planar waveguide may be described by the propagation constants $[10,11] \beta_{\mathrm{TE}} \neq \beta_{\mathrm{TM}}$. That is why after passing a path of the length $x$ between the propagating modes $\mathrm{TE}_{m}$ and $\mathrm{TM}_{m}$ the phase difference is $\Delta \phi$, defined as [9]:

$$
\Delta \phi=\left|\beta_{\mathrm{TE}_{m}}-\beta_{\mathrm{TM}_{m}}\right| \times x,
$$

where $\Delta \phi$ - phase difference between the propagating

*e-mail: kazimierz.gut@polsl.pl modes, $\beta_{\mathrm{TE} m}, \beta_{\mathrm{TM} m}-\mathrm{TE}$ and TM $m$-order mode propagation constants.

If the phase difference between the modes amounts to $2 \pi$ after passing the path of propagation $L_{\mathrm{B} m}$, it is called the beat length $m$-pair of orthogonal modes [7]:

$$
2 \pi=\left|\beta_{\mathrm{TE}_{m}}-\beta_{\mathrm{TM}_{m}}\right| \times L_{\mathrm{B}_{m}} .
$$

As

$$
\beta=2 \pi N_{\text {eff }} / \lambda,
$$

we get

$$
\left|N_{\mathrm{eff}_{\mathrm{TE}}}-N_{\mathrm{eff}_{\mathrm{TM}}}\right|=\lambda / L_{\mathrm{B}_{m}},
$$

where $N_{\text {effte }}\left(N_{\text {effTM }}\right)$ - effective refractive index mode TE (TM), $\lambda$ - wavelength, $N_{\text {effTE }}-N_{\text {effTM }}-$ modal birefringence.

The functioning of most waveguide planar sensors is based on the phenomena of differential interference and detected by measuring the phase difference $\Delta \phi$ between the propagating modes $\mathrm{TE}_{m}$ and $\mathrm{TM}_{m}$ at the end of the waveguide [12-18].

For planar waveguide structure several methods of measuring the beat length have been suggested. The effective refractive indices of modes are determined by means of a prism coupler and also their differences [19]. Another method is based on an analysis of the polarization states of the output light in response to known input polarization states [20]. When superluminescent diodes (SLD) are applied, the spectral interference method permits to determine the modal birefringence [21]. For this purpose an immersion coupler may be used which allows the light to leave from the two waveguide modes continuously and to determine the beat length [10]. If only one mode is excited and a spot excitation of displacement is applied, coupling some part of energy with the orthogonal mode, also this value can be determined [22].

\section{Determination of modal birefringence using scattered light}

Measurements of modal birefringence applying the method of recording the intensity of scattered light are absolutely reliable, non-destructive and fast in their execution [23, 24]. 
The intensity of scattered light was recorded by means of a CCD camera, provided with an optical system, which permits to focus on the surface of the waveguide. For the purpose of the determined modal birefringence the interference of scattered light may be utilized. This test stand is shown in Fig. 2. It is equipped with a clasp holding the waveguide, a CCD camera and a computer with a specially assigned software. The holder of the waveguide is provided with a manipulator which permits to adjust the position of the structure in relation to the camera. In the arm of the holder there is a laser as well as a diaphragm. The CCD camera is mounted above the upper plane of the waveguide. It possesses an optical system with an adjustable focal distance. It is of extreme importance that the camera is equipped with an adequate CCD transducer with linear sensitivity characteristics in the given range of wavelength in which the investigations are being run.

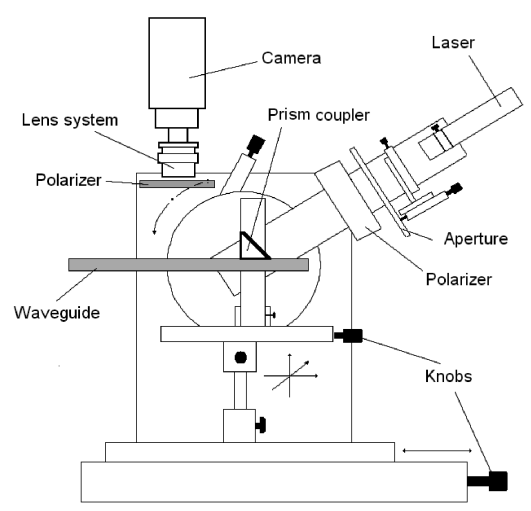

Fig. 2. Diagram of the test stand for measurements of the beat length of planar waveguides making use of scattered light.

For the purpose of introducing light into the waveguide a prism coupler was applied. By adjusting the polarization input beam appropriately, it was possible to excite the modes $\mathrm{TE}_{i}$ and $\mathrm{TM}_{i}$ simultaneously. A definite pair of modes was selected by changing the angle of the beam input to the prism. The image was detected by means of a black-and-white camera GKB(CB23803SA 1/3" IR BW CCD). Placing an additional polarizer in front of the camera we can observe oscillations of the intensity of light (in the direction of its propagation) connected with the interference of scattered light from the modes TE and TM. The recorded photo and the average distribution of the intensity of light (several horizontal lines of pixels) along the path of propagation are to be seen in Fig. 3. The planar single mode structure of the waveguide was made in a glass substrate (soda-lime) applying the ion-exchange technique $\mathrm{K}^{+}-\mathrm{Na}^{+}$.

Determining the distance between the first and the last distinct maximum (minimum) and the number of oscillations between, then we determine the beat length and can calculate the modal birefringence of the investigated waveguide ( $\lambda=635 \mathrm{~nm}$ and $L_{\mathrm{B}}=0.84 \mathrm{~mm}$, the modal birefringence amounts to $\left.7.5 \times 10^{-4}\right)$.

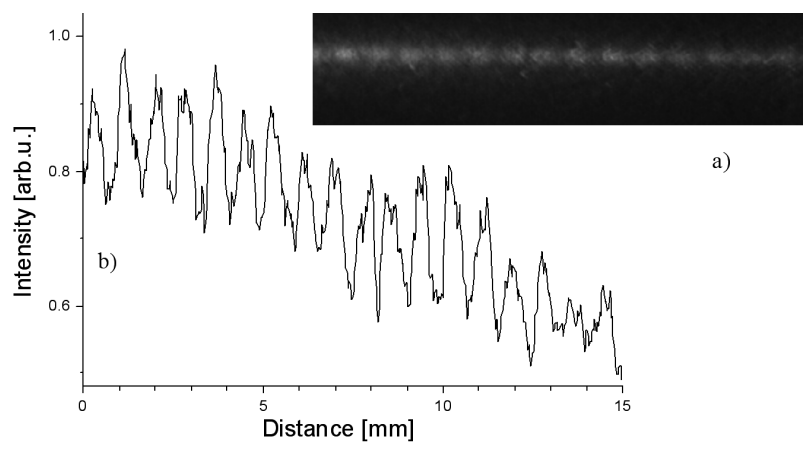

Fig. 3. (a) Image captured by the CCD camera (the polarizer was mounted in front of the camera). (b) Dependence of the intensity of scattered light. Analyzed images of excited modes $\left(\mathrm{TE}_{0}\right.$ and $\left.\mathrm{TM}_{0}\right)$.

\section{Determination of modal birefringence by means of an immersion coupler}

Figure 4 presents the measurement system for the determination of modal birefringence by means of an immersion coupler. In the investigated planar waveguide, two orthogonal modes TE and TM are excited by the same light source using an input prism coupler (PC) whose coupling is constant during the measurements. By adjusting the polarization input beam appropriately, it was possible to excite the modes TE and TM simultaneously. A definite pair of modes was selected by changing the angle of the beam input to the prism.

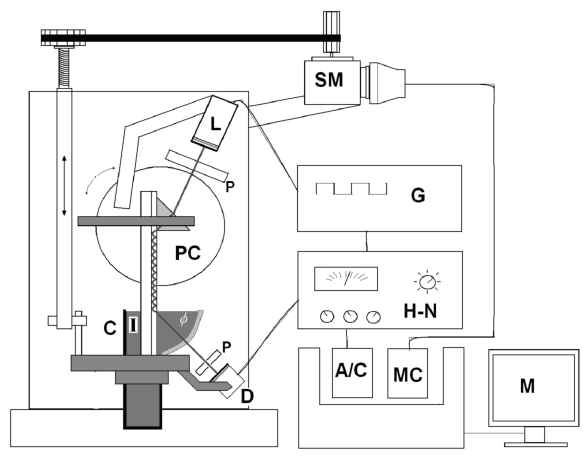

Fig. 4. Diagram of the test stand for measurements of the beat length of planar waveguides by means of an immersion coupler. SM - step motor, G - generator, $\mathrm{H}-\mathrm{N}$ - homodyne nanovoltometer, $\mathrm{A} / \mathrm{C}$ - analog to digital converter, $\mathrm{C}$ - cuvette, $\mathrm{D}$ - detector, $\mathrm{L}$ laser diode, $\mathrm{P}$ - polarizer, I - immersion liquid, PC - prism coupler, $\mathrm{MC}$ - motor card, $\mathrm{M}$ - monitor.

The source of light, a polarizer and the system prismwaveguide are joined stiffly with each other, which ensures an invariable level of power introduced to the waveguide during the measurements. The role of the output coupler was played by a cuvette filled with liquid with an appropriately high refractive index, in which the investigated waveguide were immersed. By changing the immer- 
sion depth of the investigated waveguide in the immersion liquid the propagation length of light in the waveguide changes. Due to a mismatch of the conditions of propagation along the border waveguide-immersion liquid $\left(n_{1}>N_{\text {eff }}\right.$ where $n_{1}$ - refractive index of the liquid, $N_{\text {eff }}$ - effective refractive index of the waveguide) the guided light is radiated out of the waveguide. This process takes place immediately beneath the surface of the immersion liquid. The output beams leave the cuvette passing through its transparent cylinder-shaped wall, and radiate out of the waveguide.

Such a solution ensures that the point, where the light is guided out of the waveguide, does not change its position with respect to the cuvette. In the discussed measurement system, $\alpha$-bromonaphtalene was used as the immersion liquid. By raising the cuvette the immersion depth of the waveguide increases, and accordingly the length of light propagation in the waveguide decreases [6]. On the light path coming from the modes TE and TM a polarizer $\mathrm{P}$ was placed, and the interference signal was recorded on the detector. The power recorded by the detector $\mathrm{D}$ depends on the phase difference between the beams coming from the modes TE and TM. Both the control of the system and the acquisition and processing of data were controlled by a computer.

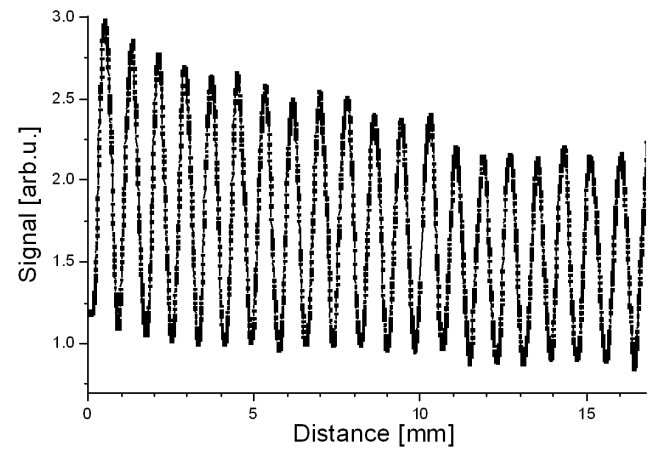

Fig. 5. Signal recorded by the detector after the change of the propagation length.

Figure 5 presents the signal recorded by the detector. By determining the length between two neighbouring maxima values, the beat length $L_{\mathrm{B}}$ can be found. Applying Eq. (4) we can determine the module of the modal birefringence. The application of an immersion coupler provided the same value of the beat length $L_{\mathrm{B}}$ as in the case of the method described above.

\section{Conclusion}

The measurement methods presented in the paper permit to determine principally the difference of propagation constants and not only their change, as has often been presented in other publications. The presented method of taking measurements is particularly adequate when the difference of the propagation constants changes along the path of propagation.

\section{Acknowledgments}

The investigations were sponsored by the Ministry of Science and High Education of Poland within the grant O R00 017912.

\section{References}

[1] G. Konieczny, T. Pustelny, Acta Phys. Pol. A 122, 962 (2012).

[2] T. Pustelny, C. Tyszkiewicz, K. Barczak, Opt. Appl. 33, 469 (2003).

[3] K. Barczak, Bull. Pol. Acad. Sci. Techn. Sci. 59, 409 (2011).

[4] T. Pustelny, J. Ignac-Nowicka, Z. Opilski, Opt. Appl. 34, 563 (2004).

[5] K. Barczak, T. Pustelny, D. Dorosz, J. Dorosz, Europ. Phys. J. ST 154, 11 (2008).

[6] T. Pustelny, M. Procek, E. Maciak, A. Stolarczyk, S. Drewniak, M. Urbańczyk, M. Setkiewicz, K. Gut, Z. Opilski, Bull. Pol. Acad. Sci., Tech. Sci. 60,853 (2012).

[7] B. Beche, N. Pelletier, E. Gaviot, J. Zyss, Opt. Commun. 230, 91 (2004).

[8] R. Rogozinski, K. Gut, P. Karasiński, Z. Opilski, A. Opilski, Proc. SPIE 3581, 375 (1998).

[9] K. Gut, A. Zakrzewski, T. Pustelny, Acta Phys. Pol. A 118, 1140 (2010).

[10] K. Gut, Proc. SPIE 5956, 59561N-1 (2005).

[11] M. Szczurowski, W. Urbanczyk, M. Napiorkowski, P. Hlubina, U. Hollenbach, H. Sieber, J. Mohr, Appl. Opt. 50, 2594 (2011).

[12] A. Opilski, R. Rogoziński, K. Gut, M. Błahut, Z. Opilski, Opto-Electron. Rev. 8, 117 (2000).

[13] K. Gut, Acta Phys. Pol. A 114, A121 (2008).

[14] K. Gut, S. Drewniak, Acta Phys. Pol. A 118, 1133 (2010).

[15] K. Gut, T. Pustelny, D. Nabaglo, Acta Phys. Pol. A 118, 1136 (2010).

[16] K. Gut, P. Karasiński, W.T. Wójcik, R. Rogoziński, Z. Opilski, A. Opilski, Opt. Appl. 29, 101 (1999).

[17] K. Gut, S. Drewniak, Acta Phys. Pol. A 120, 630 (2011).

[18] K. Gut, Bull. Pol. Acad. Sci. Techn. Sci. 59, 395 (2011).

[19] S.Y. Cheng, K.S. Chiang, H.P Chan, Phot. Tech. Lett. 15, 700 (2003).

[20] Z.B. Ren, Ph. Robert, P.A. Paratte, J. Phys. E, Sci. Instrum. 18, 859 (1985).

[21] G. Golojuch, U. Hollenbach, T. Mappes, J. Mohr, A. Urbanczyk, W. Urbanczyk, Meas. Sci. Technol. 19, 025304 (2008).

[22] W.W. Hu, K. Inagaki, Y. Mizuguchi, Opt. Lett. 26, 193 (2001).

[23] K. Gut, Acta Phys. Pol. A 122, 818 (2012).

[24] K. Gut, K. Nowak, Eur. Phys. J. ST 154, 89 (2008). 